\title{
Caracterização física e química de frutos de umbugueleira (Spondias sp.) no
}

\section{semiárido pernambucano}

\author{
Physical and chemical characterization of umbugueleira fruits (Spondias sp.) in the semi-arid state \\ of Pernambuco
}

Caracterización física y química de frutos de umbugueleira (Spondias sp.) en estado semiárido de Pernambuco

\section{Resumo}

As frutas nativas passaram a ser vistas como uma excelente alternativa para a região Nordeste, especialmente a umbugueleira. Os frutos das Spondias sp. são apreciados pelos consumidores devido aos compostos bioativos, o que tem favorecido agregar valor aos produtos e aumentar a renda familiar. No entanto, pesquisas sobre qualidade de frutos são necessárias e importantes, já que os resultados obtidos são ainda inconsistentes. Face ao exposto, objetivouse neste trabalho avaliar as características físicas e químicas de frutos de umbugueleira no semiárido pernambucano. O trabalho foi realizado no Laboratório de Química da Unidade Acadêmica de Serra Talhada da Universidade Federal Rural de Pernambuco. Utilizou-se fruto no estádio maduro, com coloração da casca amarela. Foram selecionadas quanto à uniformidade, em seguida, realizou-se a caracterização física e química da massa do fruto, formato do fruto, rendimento de polpa, casca e de semente, teor de sólidos solúveis ( ${ }^{\circ}$ Brix), acidez titulável, $\mathrm{pH}$, e o índice de maturação (relação SS/AT), respectivamente. Utilizou-se 20 repetições de 10 frutos. Os dados foram obtidos por meio de parâmetros descritivos e determinação dos coeficientes de correlação linear de Pearson. Com base nos resultados, observou-se que os frutos com maior comprimento e diâmetro obtiveram maior rendimento de polpa. A relação SS/AT apresentou maior correlação com os sólidos solúveis do que a acidez dos frutos, sendo mais representativa que a medição isolada desses parâmetros, uma vez que o índice de maturação é uma das formas mais adequadas para avaliar o sabor.

Palavras-chave: Fruteira; Pós-colheita; Umbuguela; Spondias sp.

\begin{abstract}
Native fruits came to be seen as an excellent alternative to the Northeast region, especially the umbugueleira. The fruits of spondias sp. are appreciated by consumers due to bioactive compounds, which has favored adding value to products and increasing family income. However, research on fruit quality is necessary and important, since the results obtained are still inconsistent. In view of the above, the objective of this work was to evaluate the physical and chemical characteristics of umbugueleira fruits in the semiarid state of Pernambuco. The work was carried out at the Chemistry Laboratory of the Serra Talhada Academic Unit of the Federal Rural University of Pernambuco. Fruit was used in the mature stage, with yellow peel coloration. The physical and chemical characterization of fruit mass, fruit shape, pulp yield, peel and seed, soluble solids (oBrix), titratable acidity, $\mathrm{pH}$, and maturation index (SS/TA ratio), respectively, were selected. Twenty replicates of 10 fruits were used. The data were obtained through descriptive parameters and determination of Pearson's linear correlation coefficients. Based on the results, it was observed that the fruits with higher length and diameter had higher pulp yield. The SS/TA ratio showed a higher correlation with
\end{abstract}


soluble solids than fruit acidity, being more representative than the isolated measurement of these parameters, since the maturation index is one of the most appropriate ways to evaluate the flavor.

Keywords: Fruit tree; Post-harvest; Umbuguela; Spondias sp.

\begin{abstract}
Resumen
Las frutas nativas llegaron a ser vistas como una excelente alternativa a la región noreste, especialmente la umbugueleira. Los frutos de spondias sp. son apreciados por los consumidores debido a los compuestos bioactivos, lo que ha favorecido agregar valor a los productos y aumentar los ingresos familiares. Sin embargo, la investigación sobre la calidad de la fruta es necesaria e importante, ya que los resultados obtenidos siguen siendo inconsistentes. En vista de lo anterior, el objetivo de este trabajo fue evaluar las características físicas y químicas de los frutos de umbugueleira en estado semiárido de Pernambuco. El trabajo se realizó en el Laboratorio de Química de la Unidad Académica Serra Talhada de la Universidad Federal Rural de Pernambuco. La fruta se utilizó en la etapa madura, con coloración de cáscara amarilla. Se seleccionó la caracterización física y química de la masa del fruto, la forma del fruto, el rendimiento de la pulpa, la cáscara y la semilla, los sólidos solubles (oBrix), la acidez titulable, el pH y el índice de maduración (relación SS/TA), respectivamente. Se utilizaron veinte réplicas de 10 frutas. Los datos se obtuvieron a través de parámetros descriptivos y la determinación de los coeficientes de correlación lineal de Pearson. Con base en los resultados, se observó que los frutos con mayor longitud y diámetro tenían mayor rendimiento de pulpa. La relación SS/TA mostró una mayor correlación con sólidos solubles que con la acidez de la fruta, siendo más representativa que la medición aislada de estos parámetros, ya que el índice de maduración es una de las formas más adecuadas de evaluar el sabor.
\end{abstract}

Palabras clave: Fruto; Post-cosecha; Umbuguela; Spondias sp.

\title{
1. Introdução
}

Muitas espécies frutíferas nativas já são conhecidas e exploradas economicamente, enquanto que outras necessitam de pesquisas voltadas para a cadeia produtiva e qualidade de frutos, vislumbrando nichos diversos de mercado para frutas frescas e secas, como para produtos e subprodutos (Silva Junior et al., 2021). Dentre as espécies que produzem frutos com grandes possibilidades de utilização, pode-se mencionar a umbugueleira (Spondias sp.), pertencente à família Anacardeaceae e gênero Spondias, embora subexplorada, têm grande potencial de utilização, especialmente na agricultura familiar.

A origem desta espécie ainda é incerta e são poucos os exemplares encontrados no Nordeste, como na Paraíba e Ceará, nos municípios de Santa Isabel e Tururu, respectivamente (Souza, 1998), assim como no estado de Pernambuco, no município de Serra Talhada. De acordo com Souza (1998) e Silva et al. (2004), a origem desta espécie foi resultante, provavelmente de hibridação natural entre o umbuzeiro (S. tuberosa Arr. Cam.) e a cirigueleira (S. purpurea L.) Enquanto, Santos \& Oliveira (2008) baseado em estudo de inter-relações genéticas com base em marcadores AFLP entre espécies do gênero Spondias, sugeriram que a umbuguela possui similaridade molecular superior a $60 \%$ entre o umbuzeiro e a cajazeira $(S$. mombin).

No Nordeste, as Spondias passaram a ser vistas como uma excelente alternativa econômica devido a crescente comercialização de frutos, o que tem favorecido renda extra para os agricultores (Souza, 1998; Drummond et al., 2000; B atista et al., 2015). Fato reportado por Ataíde et al. (2020), que o desenvolvimento sustentável das comunidades rurais tem sido prioridade, principalmente com frutas nativas, favorecendo a inclusão socioprodutiva, especialmente a geração de renda familiar dos nordestinos.

Dentre as Spondias com excelente potencial a serem exploradas economicamente, a cirigueleira (Spondias purpurea L.), originária da América Tropical, possui fruto tipo drupa, coloração vermelho-escura quando maduro e polpa de aroma e sabor agradável (Leon \& Shaw, 1990). Devido a excelente qualidade organoléptica, os frutos são bastante apreciados pelos consumidores do Nordeste, refletido no aumento do consumo na forma in natura e processado, com diversos produtos comercializados, o que tem proporcionado crescente interesse do cultivo comercial (Sacramento \& Souza, 2000). Como observado para a cultura do umbuzeiro (Spondias tuberosa Arruda Câmara), com fruto exótico, sabor agradável, aroma 
peculiar e fonte de compostos bioativos, o que tem despertando interesse no mercado, tanto nacional como internacional (Silva et al., 2012; Lima et al., 2015; Mertens et al., 2016). Devido ao potencial desta frutífera, Pereira et al. (2021) estudaram a caracterização morfológica de umbus de quatro municípios paraibanos a fim de identificar genótipos promissores para consumo in natura e emprego agroindustrial. Constataram variabilidade genética nos materiais e verificaram que os frutos de Queimadas e São Vicente do Seridó apresentaram melhores caracteres morfológicos para massa, comprimento, diâmetro, matéria fresca e o rendimento dos frutos promissores para indústria.

As principais regiões produtoras do umbu situam-se no estado da Bahia, com comercialização em feiras livres na forma de fruta fresca e para processamento de polpa (Ataíde et al., 2017). Utilizado na fabricação de cerveja artesanal, além de cultivos comerciais neste estado vêm sendo incrementados com novas áreas de produtores orgânicos, visando agregar valor aos produtos e aumentar a renda familiar (Ataíde et al., 2020).

Apesar de a umbuguela apresentar grandes possibilidades de utilização e ser bastante apreciada para o consumo, principalmente in natura, no entanto, pesquisas voltadas para a qualidade de frutos são ainda necessárias, já que os resultados obtidos por muitos autores são ainda divergentes. De acordo com Santos et al (2010), muitos resultados de pesquisas, principalmente de caracterização físicas e químicas de frutos de determinadas espécies variam, tanto pelo potencial genético, local ou região de cultivo, práticas culturais adotadas, época de colheita, estádio de maturação, entre outros fatores. Como observado também pela combinação entre porta enxertos e seleções de laranjeiras, influenciando tanto o teor de sólidos solúveis, acidez titulável, como a massa, comprimento e diâmetro dos frutos (Bastos et al., 2021). Face ao exposto, objetivouse neste trabalho avaliar as características físicas e químicas de frutos de umbugueleira no semiárido pernambucano.

\section{Metodologia}

O trabalho foi realizado no laboratório de Química da Unidade Acadêmica de Serra Talhada, pertencente à Universidade Federal Rural de Pernambuco (UAST/UFRPE), município de Serra Talhada, PE, situado no Sertão Pernambucano, Alto do Pajeú. A região situa-se nas coordenadas de 0759'31" de latitude e 38¹7'54" de longitude, com altitude média de $530 \mathrm{~m}$. O clima da região é semiárido, com chuvas concentradas nos meses de março e abril, precipitação média anual de $550 \mathrm{~mm}$, temperatura média de $32{ }^{\circ} \mathrm{C}$, umidade relativa do ar de 62,8\% (Neto et al., 2011).

Os frutos foram colhidos do pomar da UAST/UFRPE no estádio de maturação maduro 7 (Figura 1), acondicionaramse em recipientes plásticos e transportados para o laboratório, e selecionados quanto ao estádio de maturação e uniformidade. Posteriormente avaliados os seguintes atributos físicos: massa média dos frutos, aferidos com auxílio de balança analítica digital com precisão de $0,001 \mathrm{~g}$, resultados em $(\mathrm{g})$; rendimento da polpa (\%), calculada pela diferença entre a massa do fruto e a massa da polpa e obtido em porcentagem (\%), rendimento da casca (\%), obtido através da equação: $\mathbf{R}=$ peso da casca / peso total do fruto x 100 e rendimento das sementes, determinado através da equação: $\mathbf{R}=$ massa das sementes / peso total do fruto x 100; comprimento e diâmetro do fruto $(\mathrm{cm})$, mensurados com paquímetro digital, e formato do fruto, obtido mediante a relação entre o comprimento e o diâmetro do fruto. Relação < 1 - frutos achatados, relação = 1 - frutos arredondados e relação $>1$ - frutos alongados.

As características químicas das umbuguelas foram analisadas da polpa homogenizada com auxílio de um 'mixer', mediante aferição do teor de sólidos solúveis (SS), determinado por meio da leitura refratométrica direta em graus Brix ( $\left.{ }^{\circ} B r i x\right)$ de uma alíquota da polpa homogeneizada, em refratômetro digital tipo Palette PR - 32, marca ATAGO, com compensação de temperatura automática, segundo critérios da AOAC (2005); acidez titulável (AT), obtida de acordo com as Normas Analíticas do Instituto Adolfo Lutz (2008), em que utilizaram-se $5 \mathrm{~g}$ de polpa homogeneizada em triturador doméstico tipo 'mixer' e 
diluída em $95 \mathrm{~mL}$ de água destilada, seguida da titulação com solução padronizada de $\mathrm{NaOH}$ a $0,1 \mathrm{~N}$, tendo como indicador o ponto de viragem da fenolftaleína. Os resultados foram expressos em porcentagem, correspondente a gramas (g) de ácido cítrico $100 \mathrm{~g}^{-1}$ da amostra; $\mathrm{pH}$, mensurado em polpa homogeneizada dos frutos, triturados com auxílio de um 'mixer'. Utilizouse o potenciômetro, conforme os procedimentos recomendados pela AOAC (2005); e o índice de maturação (SS/AT), calculado através da relação entre os sólidos solúveis (SS) e a acidez titulável (AT).

Para a determinação da qualidade através da caracterização física e química, as umbuguelas foram distribuídas em 20 repetições de 10 frutos cada. Os dados foram tabelados e calcularam-se as medias e analisados por meio dos parâmetros estatísticos descritivos e a determinação dos coeficientes de correlação linear de Pearson.

Figura 1: Estádios de maturação (de 1 a 7) de frutos da umbugueleira.

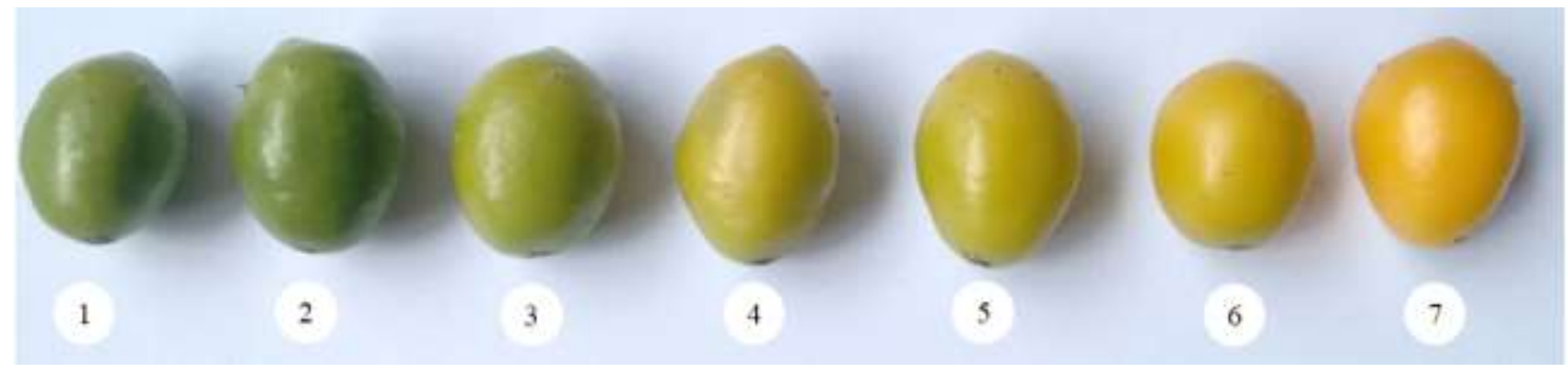

Fonte: Arquivo pessoal, Grupo de Estudo Fruticultura Rural (GEFRUTRURAL, 2021).

\section{Resultados e Discussão}

Os resultados obtidos para as características físicas dos frutos de umbugueleira, verificou-se que a massa fresca média da umbuguela no estádio de maturação maduro 7 (Figura 1) foi de 7,54 g (Tabela 1). Resultados semelhantes foram encontrados por Dantas et al. (2016), estudando o desenvolvimento, fisiologia e indicadores do ponto de colheita de frutos de umbugueleira, com média obtida para massa do fruto de 10,63 g. Geralmente a massa de umbuguela é menor em relação ao umbu, cajá e seriguela no estádio de maturação plena, com valores médios de 21g, 11,92g e 13,98g, respectivamente (Pereira et al., 2021; Carvalho et al., 2016; Martins et al., 2003). De acordo com Carvalho et al. (2003), os valores das características físicas e químicas de frutos dependem das condições edafoclimáticas do cultivo, constituição genética da planta, tratos culturais e estádio de maturação do fruto.

Para Carvalho et al. (2011) e Silvino et al. (2017), a massa do fruto é um atributo importante para o mercado tanto para frutas frescas, como para processadas, quanto maior a massa e tamanho do fruto, mais atrativo para o consumidor, além da maior rentabilidade para a indústria. No presente estudo, a média obtida para o comprimento e diâmetro das umbuguelas,foi de $28,66 \mathrm{~mm}$ e $21,33 \mathrm{~mm}$, respectivamente. Já o formato do fruto foi 1,32, enquadrando no formato ovoide. Resultados semelhantes foram obtidos por Dantas et al. (2016), com médias obtidas para comprimento de 29,95 mm e diâmetro 23,43 mm, e formato ovoide em frutos no estádio de maturação pleno de umbugueleiras.

Os resultados médios obtidos para o rendimento da casca, rendimento da semente, e rendimento da polpa das umbuguelas foram de 25,50\%, 13,69\% e 53,08\%, respectivamente (Tabela 1). Em relação ao rendimento de polpa e casca das umbuguelas, observa-se porcentagem média de 78,58 \%. Tal resultado aponta que a umbuguela poderá ser utilizada tanto para o consumo in natura, como para o processamento. Rendimentos semelhantes foram encontrados por Menezes et al. (2017) e Costa et al. (2015), em frutos de umbuzeiro (Spondias tuberosa) no estádio de maturação maduro, com rendimento médio de polpa de $74,30 \%$ e $65,08 \%$, respectivamente. 
Tabela 1. Médias de massa fruto $(\mathrm{MF})$, comprimento fruto $(\mathrm{CF})$, diâmetro fruto $(\mathrm{DF})$, formato do fruto $(\mathrm{FF})$, rendimento da casca (RC), rendimento da semente (RS) e rendimento de polpa (RP) de umbuguelas. Serra Talhada/PE. 2021.

\begin{tabular}{cccccccc}
\hline Fonte de variação & $\mathrm{MF}$ & $\mathrm{CF}$ & $\mathrm{DF}$ & $\mathrm{FF}$ & $\begin{array}{c}\mathrm{RC} \\
(\%)\end{array}$ & $\begin{array}{c}\mathrm{RS} \\
(\%)\end{array}$ & $\begin{array}{c}\mathrm{RP} \\
(\%)\end{array}$ \\
\hline Média & 7,54 & 28,66 & 21,33 & 1,32 & 25,50 & 13,69 & 53,08 \\
DP & 0,47 & 0,52 & 0,40 & 0,02 & 2,24 & 1,13 & 2,48 \\
CV $(\%)$ & 6,21 & 1,82 & 1,87 & 1,71 & 8,78 & 8,28 & 4,67 \\
\hline
\end{tabular}

DP: desvio padrão; CV: coeficiente de variação. Fonte: Dados de pesquisa do Grupo de Estudo Fruticultura Rural (GEFRUTRURAL, 2021).

Quando analisadas as características químicas da polpa das umbuguelas, verificou-se que o teor de sólidos solúveis foi em média $13,39^{\circ}$ Brix, enquanto a média obtida para a acidez titulável foi de 0,64\% (de ácido cítrico). Já o valor médio de pH de 2,96. Em relação ao índice de maturação, a média obtida foi de 21,04 (Tabela 2). Dantas et al. (2016) analisando umbuguelas maduras encontraram médias de sólidos solúveis de 20,68\%, acidez titulável de 0,63\%, pH de 3,33, e o índice de maturação de 35,28. Tais resultados encontrados pelos respectivos autores, estão diretamente relacionados às condições edafoclimáticas, assim como, o grau de maturação na colheita. Tais fatores influenciam diretamente estas variáveis e aos demais atributos de qualidade pós-colheita dos frutos (Chitarra \& Chitarra, 2005).

De acordo com o Padrão de Identidade e Qualidade (PIQ) do Ministério da Agricultura Pecuária e do Abastecimento (MAPA) (BRASIL, 1999), os valores de pH, sólidos solúveis e acidez titulável mínimo permitidos para polpa de cajá ( $S$. mombin) são de 2,2, 9,0\% e 0,90\%, respectivamente. Resultados obtidos no presente estudo estão em conformidade com a legislação, exceto a acidez titulável. De acordo com Santos et al. (2010), frutos com elevado teor de sólidos solúveis são preferidos tanto para o consumo in natura, como para indústria, por proporcionar maior rendimento no processamento, em função da maior quantidade e qualidade de néctar produzido pela polpa.

Outra característica importante é o índice de maturação (SS/AT) (Tabela 2), este atributo determina o sabor do suco dos frutos, sendo mais representativo do que a determinação isolada de sólidos solúveis ou acidez titulável, uma vez que o índice de maturação exprime a natureza doce-ácido do suco (Ataíde et al., 2018). Dessa forma, quanto maior os valores deste índice, mais doce é a fruta (Chitarra \& Chitarra, 2005).

Tabela 2. Médias de sólidos solúveis ( ${ }^{\circ}$ Brix) (SS), acidez titulável (\% ácido cítrico) (AT), pH, e índice de maturação da polpa (IM (SS/AT)) das umbuguelas. Serra Talhada-PE. 2021.

\begin{tabular}{ccccc}
\hline Fonte de variação & SS & AT & pH & $\begin{array}{c}\text { IM } \\
(\text { SS/AT) }\end{array}$ \\
\hline Média & 13,39 & 0,64 & 2,96 & 21,04 \\
DP & 0,63 & 0,05 & 0,05 & 2,27 \\
CV $(\%)$ & 4,69 & 8,04 & 1,75 & 10,77 \\
\hline
\end{tabular}

DP: desvio padrão; CV: coeficiente de variação. Fonte: Dados de pesquisa do Grupo de Estudo Fruticultura Rural (GEFRUTRURAL, 2021).

Na Tabela 3, podemos observar a correlação linear de Pearson entre as características físicas e físico-químicas das umbuguelas provenientes do município de Serra Talhada-PE. As correlações tanto positivas quanto negativas entre os atributos 
de qualidade de frutos indicam que estas são beneficiadas ou prejudicadas pelas mesmas causas de variação (Nascimento et al., 2014).

A massa dos frutos, sendo uma das características mais importante, apresentou correlação positiva significativa $(\mathrm{p} \leq 0,01)$ para o tamanho do fruto, sendo mais sensíveis ao comprimento do fruto $\left(0,75^{* *}\right)$, que ao diâmetro $(0,50 * *)$, apresentando uma correlação considerada de forte a média, segundo a classificação de intensidade proposta por Guerra e Liveira (1999). Tais resultados indicam que a massa dos frutos está diretamente relacionada seu comprimento e diâmetro. Para a variável rendimento de polpa com o rendimento da casca e semente, constatou-se uma correlação negativa não significativa (-0,29 ns e -0,38ns) (Tabela 3). Desse modo, os frutos que apresentaram maior rendimento de casca e semente, evidenciam menor rendimento de polpa.

Ao analisar o $\mathrm{pH}$, observa-se uma correlação negativa significativa $(\mathrm{p} \leq 0,01)$ em relação a acidez titulável $\left(-0,72^{* *}\right)$ (Tabela 3), indicando que o pH é inversamente proporcional a acidez, ou seja, a medida que o pH aumenta a acidez titulável diminui. O índice de maturação (SS/AT), apresentou maior correlação negativa com a acidez titulável (-0,90**) e positiva com o teor de sólidos solúveis $\left(0,76^{* *}\right)$, e o pH $\left(0,72^{* *}\right)$. Logo, o aumento da acidez titulável foi responsável pela redução do índice de maturação, visto que a acidez titulável é importante para definir o sabor dos frutos. Portanto, a correlação entre as características físicas e químicas é importante para programas de melhoramento genético (Silva et al., 2013).

Tabela 3. Coeficientes de correlação linear de Pearson de atributos de qualidade de umbuguelas entre massa (MF), comprimento $(\mathrm{CF})$, diâmetro $(\mathrm{DF})$, formato do fruto $(\mathrm{FF})$, rendimento da polpa (RP), rendimento da casca (RC), rendimento da semente (RS), acidez titulável (AT), pH, sólidos solúveis (SS), índice de maturação (IM (SS/AT)). Serra Talhada - PE. 2021.

\begin{tabular}{cccccccccccc}
\hline & MF & CF & DF & FF & RP & RC & RS & AT & pH & SS & IM \\
\hline MF & 1 & $0,75^{* *}$ & $0,50^{* *}$ & $0,26 \mathrm{~ns}$ & $-0,27 \mathrm{~ns}$ & $-0,27 \mathrm{~ns}$ & $-0,39 \mathrm{~ns}$ & $0,63^{* *}$ & $-0,53^{* *}$ & $-0,29 \mathrm{~ns}$ & $-0,55^{* *}$ \\
CF & & 1 & $0,58^{* *}$ & $0,44^{*}$ & $0,11 \mathrm{~ns}$ & $-0,25 \mathrm{~ns}$ & $-0,50^{* *}$ & $0,62^{* *}$ & $-0,53^{* *}$ & $-0,36 \mathrm{~ns}$ & $-0,64^{* *}$ \\
DF & & & 1 & $-0,48^{*}$ & $0,06 \mathrm{~ns}$ & $0,11 \mathrm{~ns}$ & $-0,18 \mathrm{~ns}$ & $0,05 \mathrm{~ns}$ & $-0,38 \mathrm{~ns}$ & $-0,10 \mathrm{~ns}$ & $-0,13 \mathrm{~ns}$ \\
FF & & & & 1 & $0,06 \mathrm{~ns}$ & $-0,39 \mathrm{~ns}$ & $-0,34 \mathrm{~ns}$ & $0,61^{* *}$ & $-0,16 \mathrm{~ns}$ & $-0,29 \mathrm{~ns}$ & $-0,55^{* *}$ \\
RP & & & & & 1 & $-0,29 \mathrm{~ns}$ & $-0,38 \mathrm{~ns}$ & $-0,02 \mathrm{~ns}$ & $-0,09 \mathrm{~ns}$ & $0,10 \mathrm{~ns}$ & $-0,01 \mathrm{~ns}$ \\
RC & & & & & & 1 & $0,32 \mathrm{~ns}$ & $-0,55^{* *}$ & $0,49^{*}$ & $0,13 \mathrm{~ns}$ & $0,47^{*}$ \\
RS & & & & & & 1 & $-0,50^{* *}$ & $0,20 \mathrm{~ns}$ & $0,17 \mathrm{~ns}$ & $0,49^{*}$ \\
AT & & & & & & & & 1 & $-0,72^{* *}$ & $-0,43 \mathrm{~ns}$ & $-0,90^{* *}$ \\
PH & & & & & & & & 1 & $0,43 \mathrm{~ns}$ & $0,72^{* *}$ \\
SS & & & & & & & & & 1 & $0,76^{* *}$ \\
IM & & & & & & & & & & 1 \\
\hline
\end{tabular}

ns = não significativo; $* *$ e $*$ significativo a $1 \%$ e $5 \%$ pelo teste t. Fonte: Dados de pesquisa do Grupo de Estudo Fruticultura Rural (GEFRUTRURAL, 2021).

\section{Conclusão}

As umbuguelas provenientes do município de Serra Talhada-PE atendem padrões para o mercado tanto de fruta fresca, devido ao tamanho atrativo do fruto para o consumidor e pelo índice de maturação, expressando sabor agradável da polpa, assim como destinado para a indústria, pelo bom rendimento de polpa e teor de sólidos solúveis, o que proporciona rendimento no processamento pela quantidade e qualidade do néctar produzido pela polpa.

A correlação linear de Pearson para os atributos de qualidade das umbuguelas apontam correlações significativas para o comprimento e diâmetro do fruto em relação a polpa e os teores de sólidos solúveis. 
Research, Society and Development, v. 10, n. 17, e236101724838, 2021 (CC BY 4.0) | ISSN 2525-3409 | DOI: http://dx.doi.org/10.33448/rsd-v10i17.24838

Os resultados obtidos permitem antever que a umbugueleira (Spondias sp.) apresenta características importantes, cuja prospecção genética deve ser priorizada, para fins de melhoramento voltado aos caracteres qualitativos.

\section{Referências}

Ataíde, E. M., Silva, M. S., Bastos, D. C. \& Souza, J. M. A. (2018). Qualidade pós-colheita de romã comercializada no semiárido pernambucano. Agrarian Academy, 4 (9), 429-436.

Ataíde, E. M., Silva, M. S., Souza, J. M. A. \& Bastos, D. C. (2017). Ácido indolbutírico e substratos no desenvolvimento de estacas de umbuzeiro em três estádios fenológicos. Agrarian Academy, 4(8), 21-33.

Ataíde, E. M., Souza, J. M. A., Bastos, D. C., Jardim, A. M. R. F. \& Costa, R. S. (2020). Ácido indolbutírico no enraizamento de estacas de umbuzeiro no estádio de repouso vegetativo. Enciclopédia Biosfera, 17(34), 1-14.

Association of Official Analytical Chemists (2005). Official methods of analysis of the association of official analytical chemists. (Ed. 11). Washington, 1015 p.

Bastos, D. C., Sombra, K. E. S., Lima, M. A. C. de, Passos, O. S., Calgaro, M. \& Ataíde, E. M. (2021). Characterization of 'Pera' orange fruits selections grafted on two rootstocks in the São Francisco Valley, Brazil. Comunicata Scientiae, 12 (3573), 1-6.

Batista, F. D. C., Silva, S., Santana, M., \& Cavalcante, A. (2015). O umbuzeiro e o semiárido brasileiro. INSA.

Brasil. Ministério da Agricultura, Pecuária e do Abastecimento. Instrução Normativa no 122, de 10 de setembro de 1999 . Diário Oficial da República Federativa do Brasil, Brasília, 13 de setembro de 1999. Seção 1.

Carvalho, A. V., Cavalcante, M. A., Santana, C. L. \& Alves, R. M. (2011). Características físicas, químicas e atividade antioxidante de frutos de matrizes de cajazeira no estado do Pará. Alimentos e Nutrição, 22(1), 45-53.

Carvalho, A. V., Chaves, R. P. F. \& Alves, R. M. (2017). Caracterização física e físico-química de frutos em matrizes de cajazeira no Estado do Pará. Embrapa Amazônia Oriental-Boletim de Pesquisa e Desenvolvimento (INFOTECA-E).

Carvalho, J. E. U., Nazaré, R. F. R. \& Oliveira, W. M. (2003). Características físicas e físico-químicas de um tipo de bacuri (Platonia insignis Mart.) com rendimento industrial superior. Revista Brasileira de Fruticultura, 25(2), 326-328.

Costa, F. R., Neder, D. G., Rego, E. R., Rego, M. M., Neder, D. G., Silva, S. M. \& Schunemann, A. P. P. (2015). Análise biométrica de frutos de umbuzeiro do semiárido brasileiro. Bioscience Journal, 31(3), 682-690.

Chitarra, M. I. F. \& Chitarra, A. B. (2005). Pós-colheita de frutas e hortaliças: fisiologia e manuseio. UFLA, 785p.

Dantas, A. L., Silva, S. D. M., Dantas, R. L., Sousa, A. S. B. D., \& Schunemann, A. P. P. (2016). Development, physiology of maturation, and indication of harvest point of umbuguela tree fruits (Spondias sp.). Revista Brasileira de Fruticultura, 38(1), 33-42.

Drumond, M. A., Kiill, L. H. P., Lima, P. C. F., Oliveira, M. C., Oliveira, V. R., Albuquerque, S. G., \& Cavalcanti, J. (2000). Estratégias para o uso sustentável da biodiversidade da caatinga. Embrapa Semiárido-Fôlder/Folheto/Cartilha (INFOTECA-E).

Guerra, N. B. \& Livera, A.V. (1999). Correlação entre o perfil sensorial e determinações físicas e químicas do abacaxi cv. 'Pérola'. Revista Brasileira de Fruticultura, 21(1), 32-35.

Instituto Adolfo Lutz - IAL (2008). Métodos físico-químicos para análise de alimentos. São Paulo: Instituto Adolfo Lutz. 2010p.

Leon, J. \& Shaw, P. E. (1990). Spondias: the red mombin and releated fruits. In: Nagy, S., Shaw, P. E. \& Wardowski, W. F. (Ed.). Fruits of tropical and subtropical origin, composition, properties and uses. Florida Science Source, 116-126.

Lima, M. S. S.; Dantas, A. C. V. L.; Fonseca, A. A. O. \& Barroso, J. P. (2015). Caracterização de frutos de genótipos selecionados de umbu-cajazeira (Spondias sp.). Interciencia, 40(5), 311-316.

Martins, L. P., Silva, S. M., Alves, R. E. \& Filgueiras, H. A. C. (2003). Desenvolvimento de Frutos de Cirigueleira (Spondias purpurea L.). Revista Brasileira de Fruticultura, 25(1), 11-14.

Menezes, P. H. S., Souza, A. A. D., Silva, E. S. D., Medeiros, R. D. D., Barbosa, N. C., \& Garcia, S. D. (2017). Influência do estádio de maturação na qualidade físico-química de frutos de umbu (Spondias tuberosa). Scientia Agropecuaria, 8(1), 73-78.

Mertens, J., germer, J., Siqueira Filho, J. A. \& Sauerborn, J. (2016). Spondias tuberosa Arruda (Anacardiaceae), a threatened tree of the Brazilian Caatinga. Brazilian Journal of Biology, 77(3), 1-11.

Nascimento, R. S. M., Cardoso, J. A., \& Cocozza, F. D. M. (2014). Caracterização física e físico-química de frutos de mangabeira (Hancornia speciosa Gomes) no oeste da Bahia. Revista Brasileira de Engenharia Agrícola e Ambiental, 18(8), p.856-860.

Neto, J. F. C., Silva, T. G. F., Araújo, J. E. M., Martins, A. J. R., Morais, J. E. F., Miranda, K. R. \& Queiroz, M. G. (2011). Caracterização das condições climáticas dos campos experimentais da Universidade Federal Rural de Pernambuco. In: XI Jornada de Ensino, Pesquisa e Extensão (JEPEX). UFRPE, CDROM. 
Research, Society and Development, v. 10, n. 17, e236101724838, 2021

(CC BY 4.0) | ISSN 2525-3409 | DOI: http://dx.doi.org/10.33448/rsd-v10i17.24838

Pereira, F. R. A., Pereira, W. E., Pessoa, A. M. D. S., \& Vasconcelos, E. S. A. G. D. (2021). Biometry in Umbu fruits from the semi-arid region of Paraiba. Revista Brasileira de Fruticultura, 43(6), (1-6).

Sacramento, C. K. \& Sousa, F. X. (2000). Cajá (Spondias mombin L.). (Série Frutas Nativas, n. 4), FUNEP, Jaboticabal. 52p.

Santos, C. A. F. \& Oliveira, V. R. (2008). Inter-relações genéticas entre espécies do gênero Spondias com base em marcadores AFLP. Revista Brasileira de Fruticultura, 30(3), 731-735.

Santos, M. B., Cardoso, R. L., Fonseca, A. A. O. \& Conceição, M. N. (2010). Caracterização e qualidade de frutos de umbu-cajá (Spondias tuberosa X S. mombin) provenientes do Recôncavo Sul da Bahia. Revista Brasileira de Fruticultura, 32(4), 1089-1097.

Silva Júnior, J. F., Souza, F. V. D. \& Pádua, J. G. (2021). A arca de Noé das frutas nativas brasileiras. Brasília, DF: Embrapa, 220p.

Silva, D. F. P., Silva, J. O. C., Matias, R. G. P., Ribeiro, M. R. \& Bruckner, C. H. (2013). Correlação entre características quantitativas e qualitativas de frutos de pessegueiros na geração F2 cultivados em região subtropical. Revista Ceres, 60(1), 53-58.

Silva, F. V. G., Silva, S. M., Silva, G. C., Mendonça, R. M. N., Alves, R. E. \& Dantas, A. L. (2012). Bioactive compounds and antioxidant activity in fruits of clone and ungrafted genotypes of yellow momb in tree. Ciência e Tecnologia de Alimentos, 32(4), 639-646.

Silva, S. M., Costa, J. P., Mendonça, R. M. N., Santos, A. F., Batista, J. L., Alves, R. E. \& Filgueiras, H. A. C. (2004). Physical and physicochemical characterization of umbuguela fruits at different maturity stages. Proceedings of the Interamerican Society for Tropical Horticulture ISTH, $47,236-237$.

Silvino, R., Silva, G., \& Santos, O. V. (2017). Qualidade nutricional e parâmetros morfológicos do fruto cajá (Spondias Mombin L.). DESAFIOS-Revista Interdisciplinar Da Universidade Federal Do Tocantins, 4(2), 3-11.

Souza, F. X. (1998). Spondias agroindustriais e os seus métodos de propagação: frutas tropicais-cajá, ciriguela, carajana, umbu, umbu-cajá e umbuguela. Embrapa Agroindustria Tropical. 